\title{
Asymptotic Behavior of Certain Integrodifferential Equations
}

\author{
Said Grace ${ }^{1}$ and Elvan Akin ${ }^{2}$ \\ ${ }^{1}$ Department of Engineering Mathematics, Cairo University, Orman, Giza 12221, Egypt \\ ${ }^{2}$ Missouri University of Science Technology, 310 Rolla Building, Rolla, MO 65409-0020, USA \\ Correspondence should be addressed to Elvan Akin; akine@mst.edu
}

Received 11 February 2016; Revised 13 May 2016; Accepted 5 June 2016

Academic Editor: Zhan Zhou

Copyright (C) 2016 S. Grace and E. Akin. This is an open access article distributed under the Creative Commons Attribution License, which permits unrestricted use, distribution, and reproduction in any medium, provided the original work is properly cited.

This paper deals with asymptotic behavior of nonoscillatory solutions of certain forced integrodifferential equations of the form: $\left(a(t) x^{\prime}(t)\right)^{\prime}=e(t)+\int_{c}^{t}(t-s)^{\alpha-1} k(t, s) f(s, x(s)) d s, c>1,0<\alpha<1$. From the obtained results, we derive a technique which can be applied to some related integrodifferential as well as integral equations.

\section{Introduction}

In this paper, we consider the integrodifferential equation

$$
\begin{aligned}
\left(a(t) x^{\prime}(t)\right)^{\prime}= & e(t) \\
& +\int_{c}^{t}(t-s)^{\alpha-1} k(t, s) f(s, x(s)) d s, \\
& c>1,0<\alpha<1 .
\end{aligned}
$$

In the sequel, we assume that

(i) $a, e \in C\left([c, \infty), \mathbb{R}^{+}\right)$;

(ii) $k \in C([c, \infty) \times[c, \infty), \mathbb{R})$ and also there exists $b \in$ $C\left([c, \infty), \mathbb{R}^{+}\right)$such that $|k(t, s)| \leq b(t)$ for all $t \geq s \geq c ;$

(iii) $f \in C([c, \infty) \times \mathbb{R}, \mathbb{R})$ and also there exist $h \in$ $C\left([c, \infty), \mathbb{R}^{+}\right)$and real numbers $\lambda, 0<\lambda \leq 1$, and $\gamma$ such that

$$
0 \leq x f(t, x) \leq t^{\gamma-1} h(t)|x|^{\lambda+1}
$$

for $x \neq 0$ and $t \geq c$.

We only consider solutions of (1) which are continuable and nontrivial in any neighborhood of $\infty$. Such a solution is said to be oscillatory if there exists a sequence $\left\{t_{n}\right\} \subset[c, \infty)$, $t_{n} \rightarrow \infty$, such that $x\left(t_{n}\right)=0$, and it is nonoscillatory otherwise.
In the last few decades, integral, integrodifferential, and fractional differential equations have gained considerable attention due to their applications in many engineering and scientific disciplines as the mathematical models for systems and processes in fields such as physics, mechanics, chemistry, aerodynamics, and the electrodynamics of complex media. For more details one can refer to [1-8].

Oscillation and asymptotic results for integral and integrodifferential equations are scarce; some results can be found in $[5,9-13]$. It seems that there are no such results for integral equations of type (1). The main objective of this paper is to establish some new criteria on the oscillatory and the asymptotic behavior of all solutions of (1). From the obtained results, we derive a technique which can be applied to some related integrodifferential as well as integral equations.

\section{Main Results}

To obtain our main results of this paper, we need the following two lemmas.

Lemma 1 (see $[5,7])$. Let $\beta, \gamma$, and $p$ be positive constants such that $p(\beta-1)+1>0$ and $p(\gamma-1)+1>0$. Then

$$
\int_{0}^{t}(t-s)^{p(\beta-1)} s^{p(\gamma-1)} d s=t^{\theta} B, \quad t \geq 0
$$

where $B:=B[p(\gamma-1)+1, p(\beta-1)+1], B[\zeta, \eta]=\int_{0}^{1} s^{\zeta-1}(1-$ $s)^{\eta-1} d s, \zeta, \eta>0$, and $\theta=p(\beta+\gamma-2)+1$. 
Lemma 2 (see [14]). If $X$ and $Y$ are nonnegative, then

$$
X^{\lambda}-(1-\lambda) Y^{\lambda}-\lambda X Y^{\lambda-1} \leq 0, \quad 0<\lambda<1,
$$

where equality holds if and only if $X=Y$.

In what follows, we let

$$
\begin{aligned}
& g_{ \pm}(t)=e(t) \pm(1-\lambda) \lambda^{\lambda /(1-\lambda)} b(t) \\
& \quad \cdot \int_{t_{1}}^{t}(t-s)^{\alpha-1} s^{\gamma-1} m^{\lambda /(\lambda-1)}(s) h^{1 /(1-\lambda)}(s) d s
\end{aligned}
$$

and $0<\lambda<1, t \geq t_{1}$ for some $t_{1} \geq c$, where $m \in$ $C\left([c, \infty), \mathbb{R}^{+}\right)$.

Now we give sufficient conditions under which any solution $x$ of (1) satisfies $|x(t)|=O\left(t^{2}\right)$ as $t \rightarrow \infty$.

Theorem 3. Let $0<\lambda<1$ and conditions (i)-(iii) hold and suppose that $p>1, q=p /(p-1), \alpha>0, \gamma=2-\alpha-1 / p$, $p(\alpha-1)+1>0, p(\gamma-1)+1>0$, and

$$
\begin{gathered}
\frac{t}{a(t)} \text { and } b(t) \text { are bounded on }[c, \infty), \\
\int_{t_{1}}^{\infty} \frac{s}{a(s)} d s<\infty, \\
\int_{t_{1}}^{\infty}\left(s^{2} m(s)\right)^{q} d s<\infty .
\end{gathered}
$$

If

$$
\begin{aligned}
& \limsup _{t \rightarrow \infty} \frac{1}{t^{2}} \int_{t_{1}}^{t} \frac{1}{a(u)} \int_{t_{0}}^{u} g_{-}(s) d s d u<\infty, \\
& \liminf _{t \rightarrow \infty} \frac{1}{t^{2}} \int_{t_{1}}^{t} \frac{1}{a(u)} \int_{t_{0}}^{u} g_{+}(s) d s d u>-\infty
\end{aligned}
$$

for any $t_{1} \geq c$, then every nonoscillatory solution $x(t)$ of (1) satisfies

$$
\limsup _{t \rightarrow \infty} \frac{|x(t)|}{t^{2}}<\infty
$$

Proof. Let $x$ be a nonoscillatory solution of (1). We may assume that $x(t)>0$ for $t \geq t_{1}$ for some $t_{1} \geq c$. We let $F(t)=f(t, x(t))$. In view of (i)-(iii) we may then write

$$
\left(a(t) x^{\prime}(t)\right)^{\prime} \leq e(t)+b(t) \int_{c}^{t}(t-s)^{\alpha-1} f(s, x(s)) d s
$$

and so

$$
\begin{aligned}
& \left(a(t) x^{\prime}(t)\right)^{\prime} \leq e(t)+b(t) \int_{c}^{t_{1}}(t-s)^{\alpha-1}|F(s)| d s \\
& \quad+b(t) \\
& \quad \cdot \int_{t_{1}}^{t}(t-s)^{\alpha-1} s^{\gamma-1}\left[h(s) x^{\lambda}(s)-m(s) x(s)\right] d s \\
& \quad+b(t) \int_{t_{1}}^{t}(t-s)^{\alpha-1} s^{\gamma-1} m(s) x(s) d s .
\end{aligned}
$$

Applying (4) of Lemma 2 to $h(s) x^{\lambda}(s)-m(s) x(s)$ with $X=$ $h^{1 / \lambda} x$ and $Y=\left((1 / \lambda) m h^{-1 / \lambda}\right)^{1 /(\lambda-1)}$ we have

$$
\begin{aligned}
h(s) & x^{\lambda}(s)-m(s) x(s) \\
\quad \leq & (1-\lambda) \lambda^{\lambda /(1-\lambda)} m^{\lambda /(\lambda-1)}(s) h^{1 /(1-\lambda)}(s),
\end{aligned}
$$

and hence we obtain

$$
\begin{aligned}
& \left(a(t) x^{\prime}(t)\right)^{\prime} \leq e(t)+b(t) \int_{c}^{t_{1}}(t-s)^{\alpha-1}|F(s)| d s \\
& +(1-\lambda) \lambda^{\lambda /(1-\lambda)} b(t) \\
& \cdot \int_{t_{1}}^{t}(t-s)^{\alpha-1} s^{\gamma-1} m^{\lambda /(\lambda-1)}(s) h^{1 /(1-\lambda)}(s) d s+b(t) \\
& \cdot \int_{t_{1}}^{t}(t-s)^{\alpha-1} s^{\gamma-1} m(s) x(s) d s
\end{aligned}
$$

or

$$
\begin{aligned}
\left(a(t) x^{\prime}(t)\right)^{\prime} \leq & b(t) \int_{c}^{t_{1}}\left(t_{1}-s\right)^{\alpha-1}|F(s)| d s+g_{+}(t) \\
& +b(t) \int_{t_{1}}^{t}(t-s)^{\alpha-1} s^{\gamma-1} m(s) x(s) d s \\
\leq & C_{1}+g_{+}(t) \\
& +k_{1} \int_{t_{1}}^{t}(t-s)^{\alpha-1} s^{\gamma-1} m(s) x(s) d s,
\end{aligned}
$$

where $C_{1}$ and $k_{1}$ are the upper bounds of the functions $b(t) \int_{a}^{t_{1}}\left(t_{1}-s\right)^{\alpha-1}|F(s)| d s$ and $b(t)$, respectively. Integrating inequality (15) from $t_{1}$ to $t$ we have

$$
\begin{aligned}
x^{\prime}(t) \leq & \frac{a\left(t_{1}\right) x^{\prime}\left(t_{1}\right)}{a(t)}+\frac{C_{1}\left(t-t_{1}\right)}{a(t)} \\
& +\frac{1}{a(t)} \int_{t_{1}}^{t} g_{+}(s) d s \\
& +\frac{k_{1}}{a(t)} \int_{t_{1}}^{t} \int_{t_{1}}^{u}(u-s)^{\alpha-1} s^{\gamma-1} m(s) x(s) d s d u .
\end{aligned}
$$

Interchanging the order of integration in the last integral, we have

$$
\begin{aligned}
x^{\prime}(t) \leq & \frac{a\left(t_{1}\right) x^{\prime}\left(t_{1}\right)}{a(t)}+\frac{C_{1}\left(t-t_{1}\right)}{a(t)} \\
& +\frac{1}{a(t)} \int_{t_{1}}^{t} g_{+}(s) d s \\
& +k_{2} \int_{t_{1}}^{t}(t-s)^{\alpha} s^{\gamma-1} m(s) x(s) d s,
\end{aligned}
$$


where $k_{2}$ is the upper bound of the function $k_{1} / \alpha a(t)$. Integrating (17) from $t_{1}$ to $t$ and interchanging the order of integration in the last integral we find

$$
\begin{aligned}
x(t) \leq & x\left(t_{1}\right)+a\left(t_{1}\right) x^{\prime}\left(t_{1}\right) \int_{t_{1}}^{t} \frac{1}{a(s)} d s \\
& +\int_{t_{1}}^{t} \frac{C_{1}\left(s-t_{1}\right)}{a(s)} d s+\int_{t_{1}}^{t} \frac{1}{a(u)} \int_{t_{1}}^{u} g_{+}(s) d s d u \\
& +\frac{k_{2}}{\alpha+1} \int_{t_{1}}^{t}(t-s)^{\alpha+1} m(s) x(s) d s .
\end{aligned}
$$

Now, one can easily see that

$$
\begin{aligned}
x(t) \leq & x\left(t_{1}\right)+a\left(t_{1}\right) x^{\prime}\left(t_{1}\right) \int_{t_{1}}^{t} \frac{1}{a(s)} d s \\
& +\int_{t_{1}}^{t} \frac{C_{1}\left(s-t_{1}\right)}{a(s)} d s+\int_{t_{1}}^{t} \frac{1}{a(u)} \int_{t_{1}}^{u} g_{+}(s) d s d u \\
& +\frac{k_{2}}{\alpha+1} t^{2} \int_{t_{1}}^{t}(t-s)^{\alpha-1} m(s) x(s) d s
\end{aligned}
$$

or

$$
z(t):=\frac{x(t)}{t^{2}} \leq 1+C+k \int_{t_{1}}^{t}(t-s)^{\alpha-1} m(s) x(s) d s,
$$

where $C$ is the upper bound of the function

$$
\begin{aligned}
& \frac{1}{t^{2}}\left[x\left(t_{1}\right)+a\left(t_{1}\right) x^{\prime}\left(t_{1}\right) \int_{t_{1}}^{t} \frac{1}{a(s)} d s\right. \\
& \left.\quad+\int_{t_{1}}^{t} \frac{C_{1}\left(s-t_{1}\right)}{a(s)} d s+\int_{t_{1}}^{t} \frac{1}{a(u)} \int_{t_{1}}^{u} g_{+}(s) d s d u\right]
\end{aligned}
$$

and $k=k_{2} /(\alpha+1)$. Applying Holder's inequality and Lemma 1 we obtain

$$
\begin{aligned}
& \int_{t_{1}}^{t}(t-s)^{\alpha-1} s^{\gamma-1} m(s) x(s) d s \\
& \leq\left(\int_{t_{1}}^{t}(t-s)^{p(\alpha-1)} s^{p(\gamma-1)} d s\right)^{1 / p} \\
& \cdot\left(\int_{t_{1}}^{t} m^{q}(s) x^{q}(s) d s\right)^{1 / q} \\
& \leq\left(\int_{0}^{t}(t-s)^{p(\alpha-1)} s^{p(\gamma-1)} d s\right)^{1 / p} \\
& \cdot\left(\int_{t_{1}}^{t} m^{q}(s) x^{q}(s) d s\right)^{1 / q} \leq\left(B t^{\theta}\right)^{1 / p} \\
& \cdot\left(\int_{t_{1}}^{t} m^{q}(s) x^{q}(s) d s\right)^{1 / q},
\end{aligned}
$$

where $B=B[p(\gamma-1)+1, p(\alpha-1)+1]$, and $\theta=p(\alpha+\gamma-2)+1=$ 0 and so

$$
\begin{aligned}
& \int_{t_{1}}^{t}(t-s)^{\alpha-1} s^{\gamma-1} m(s) x(s) d s \\
& \quad \leq B^{1 / p}\left(\int_{t_{1}}^{t} m^{q}(s) x^{q}(s) d s\right)^{1 / q} .
\end{aligned}
$$

Thus, inequality (20) becomes

$$
z(t):=\frac{x(t)}{t^{2}} \leq C+k B^{1 / p}\left(\int_{t_{1}}^{t} m^{q}(s) x^{q}(s) d s\right)^{1 / q} .
$$

Using (24) and the elementary inequality

$$
(x+y)^{q} \leq 2^{q-1}\left(x^{q}+y^{q}\right), \quad x, y \geq 0, q>1,
$$

we obtain from (24)

$$
\begin{aligned}
& z^{q}(t) \\
& \quad \leq 2^{q-1}\left((1+C)^{q}+k^{q} B^{q / p} \int_{t_{1}}^{t} s^{2 q} m^{q}(s) z^{q}(s) d s\right) .
\end{aligned}
$$

If we denote $u(t)=z^{q}(t)$, that is, $z(t)=u^{1 / q}(t), P=2^{q-1}(1+$ $C)^{q}$, and $Q=2^{q-1} k^{q} B^{q / p}$, then

$$
u(t) \leq P+Q \int_{t_{1}}^{t} s^{2 q} m^{q}(s) u(s) d s, \quad t \geq t_{1} \geq c .
$$

The conclusion follows from Gronwall's inequality and we conclude that

$$
\limsup _{t \rightarrow \infty} \frac{x(t)}{t^{2}}<\infty
$$

If $x$ is eventually negative, we can set $y=-x$ to see that $y$ satisfies (1) with $e(t)$ being replaced by $-e(t)$ and $f(t, x)$ by $-f(t,-y)$. It follows in a similar manner that

$$
\limsup _{t \rightarrow \infty} \frac{-x(t)}{t^{2}}<\infty .
$$

From (28) and (29) we get (10). This completes the proof.

Next, by employing Theorem 3 we present the following oscillation result for (1).

Theorem 4. Let $0<\lambda<1$ and conditions (i)-(iii), (6)-(9) hold and suppose that $p>1, q=p /(p-1), \alpha>0, \gamma=$ $2-\alpha-1 / p, p(\alpha-1)+1>0$, and $p(\gamma-1)+1>0$. If for every $M, 0<M<1$,

$$
\begin{aligned}
& \limsup _{t \rightarrow \infty}\left[M t^{2}+\int_{t_{1}}^{t} \frac{1}{a(u)} \int_{t_{1}}^{u} g_{-}(s) d s d u\right]=\infty, \\
& \liminf _{t \rightarrow \infty}\left[M t^{2}+\int_{t_{1}}^{t} \frac{1}{a(u)} \int_{t_{1}}^{u} g_{+}(s) d s d u\right]=-\infty
\end{aligned}
$$

for all $t_{1} \geq c$, then (1) is oscillatory. 
Proof. Let $x$ be a nonoscillatory solution of (1), say $x(t)>0$, for $t \geq t_{1}$ for some $t_{1} \geq 0$. The proof when $x$ is eventually negative is similar. Proceeding as in the proof of Theorem 3 we arrive at (19). Therefore,

$$
\begin{aligned}
x(t) \leq & x\left(t_{1}\right)+a\left(t_{1}\right) x^{\prime}\left(t_{1}\right) \int_{t_{1}}^{\infty} \frac{1}{a(s)} d s \\
& +\int_{t_{1}}^{\infty} \frac{C_{1}\left(s-t_{1}\right)}{a(s)} d s \\
& +\int_{t_{1}}^{t} \frac{1}{a(u)} \int_{t_{1}}^{u} g_{+}(s) d s d u \\
& +k t^{2}\left(\int_{t_{1}}^{\infty} s^{2 q} m^{q}(s)\left(\frac{x(s)}{s^{2}}\right)^{q} d s\right)^{1 / q} .
\end{aligned}
$$

Clearly, the conclusion of Theorem 3 holds. This together with (7) and (8) implies that the first, second, and fourth integrals on the above inequality are bounded and hence one can easily see that

$$
x(t) \leq M_{1}+M t^{2}+\int_{t_{1}}^{t} \frac{1}{a(u)} \int_{t_{1}}^{u} g_{+}(s) d u,
$$

where $M_{1}$ and $M$ are positive constants. Note that we make $M<1$ possible by increasing the size of $t_{1}$. Finally, taking $\lim$ inf in (32) as $t \rightarrow \infty$ as well as using (30) result is a contradiction with the fact that $x$ is eventually positive.

The following corollary is immediate.

Corollary 5. Let $0<\lambda<1$ and conditions (i)-(iii), (6)-(9) hold for some $t_{1} \geq c$. In addition, assume that

$$
\begin{aligned}
& \limsup _{t \rightarrow \infty} \frac{1}{t^{2}} \int_{t_{1}}^{t} \frac{1}{a(u)} \int_{t_{1}}^{u} e(s) d s d u>\infty, \\
& \liminf _{t \rightarrow \infty} \frac{1}{t^{2}} \int_{t_{1}}^{t} \frac{1}{a(u)} \int_{t_{1}}^{u} e(s) d s d u>-\infty, \\
& \lim _{t \rightarrow \infty} \frac{1}{t^{2}} \int_{t_{1}}^{t} \frac{1}{a(u)} \int_{t_{1}}^{u}(t-s)^{\alpha-1} s^{\gamma-1} m^{\lambda /(\lambda-1)}(s) \\
& \cdot h^{1 /(1-\lambda)}(s) d s d u<\infty .
\end{aligned}
$$

If for every $M, 0<M<1$,

$$
\begin{aligned}
& \limsup _{t \rightarrow \infty}\left[M t^{2}+\int_{t_{1}}^{t} \frac{1}{a(u)} \int_{t_{1}}^{u} e(s) d s d u\right]=\infty, \\
& \liminf _{t \rightarrow \infty}\left[M t^{2}+\int_{t_{1}}^{t} \frac{1}{a(u)} \int_{t_{1}}^{u} e(s) d s d u\right]=-\infty,
\end{aligned}
$$

for all $t_{1}>c$, then (1) is oscillatory.

The following example is illustrative.
Example 6. Let $p>1,0<\alpha=1-1 / 2 p<1, \alpha=\gamma$, and $q=p /(p-1)$. Clearly,

$$
\begin{aligned}
p(\alpha-1)+1 & =p(\gamma-1)+1=p\left(1-\frac{1}{2 p}-1\right)+1 \\
& =\frac{1}{2}>0 \\
\theta & =p(\alpha+\gamma-2)+1=0 .
\end{aligned}
$$

Let the functions $a(t)$ and $b(t)$ be as in (i) and (ii) with $b(t)$ being a bounded function and let $a(t)=e^{t}, e(t)=t e^{t} \sin t$, and $f(t, x)=t^{\gamma-1} h(t) x^{\lambda}$, where $0<\lambda<1, h \in C\left(\mathbb{R}^{+}, \mathbb{R}^{+}\right)$with $h(t)=m(t), \int^{\infty} s^{2 q} h^{q}(s) d s<\infty$, and

$$
\limsup _{t \rightarrow \infty} \frac{1}{t^{2}} \int_{t_{1}}^{t} e^{-u} \int_{t_{1}}^{u}(u-s)^{\alpha-1} s^{\gamma-1} h(s) d s d u<\infty .
$$

Condition (34) is also fulfilled. Thus, all conditions of Theorem 3 are satisfied and hence every nonoscillatory solution $x$ of (1) satisfies $\lim \sup _{t \rightarrow \infty}\left(|x(t)| / t^{2}\right)<\infty$.

Now if $e(t)=t^{\delta} e^{t} \sin t, \delta \geq 2$, we see that all the hypotheses of Corollary 5 are satisfied and hence (1) is oscillatory.

Similar reasoning to that in the sublinear case guarantees the following theorems for the integrodifferential equation (1) when $\lambda=1$.

Theorem 7. Let $\lambda=1$ and the hypotheses of Theorems 3 and 4 hold with $m(t)=h(t)$ and $g_{ \pm}=e(t)$. Then the conclusion of Theorems 3 and 4 holds, respectively.

From the obtained results, we apply the employed technique to some related integrodifferential equations.

Now, we consider the integrodifferential equation

$$
\begin{array}{r}
x^{\prime}(t)=e(t)+\int_{c}^{t}(t-s)^{\alpha-1} k(t, s) f(s, x(s)) d s, \\
c>1, \alpha \in(0,1) .
\end{array}
$$

We will give sufficient conditions under which any nonoscillatory solution $x$ of (37) satisfies $|x(t)|=O(t)$ as $t \rightarrow \infty$.

Theorem 8. Let $0<\lambda<1$ and let condition (ii) hold and suppose that $p>1, q=p /(p-1), 0<\alpha<1$, and $\gamma=$ $2-\alpha-1 / p, p(\alpha-1)+1>0$, and $p(\gamma-1)+1>0$,

$$
\int_{t_{1}}^{\infty} s^{q} m^{q}(s) d s<\infty
$$

$$
\begin{aligned}
& \limsup _{t \rightarrow \infty} \frac{1}{t} \int_{c}^{t} g_{-}(s) d s<\infty, \\
& \liminf _{t \rightarrow \infty} \frac{1}{t} \int_{c}^{t} g_{+}(s) d s>-\infty
\end{aligned}
$$

for any $t_{1} \geq c$. If $x$ is a nonoscillatory solution of (37), then

$$
\limsup _{t \rightarrow \infty} \frac{|x(t)|}{t}<\infty
$$


Proof. Let $x$ be a nonoscillatory solution of (37). We may assume that $x(t)>0$ for $t \geq t_{1}$ for some $t_{1} \geq c$. We let $F(t)=f(t, x(t))$. In view of (ii) we may then write

$$
\begin{aligned}
& x^{\prime}(t) \\
& \leq e(t)+\int_{c}^{t_{1}}(t-s)^{\alpha-1}|F(s)| d s \\
& \quad+\int_{t_{1}}^{t}(t-s)^{\alpha-1} s^{\gamma-1}\left[h(s) x^{\lambda}(s)-m(s) x(s)\right] d s \\
& \quad+\int_{t_{1}}^{t}(t-s)^{\alpha-1} s^{\gamma-1} m(s) x(s) d s .
\end{aligned}
$$

Proceeding as in the proof of Theorem 3, we obtain

$$
\begin{gathered}
x^{\prime}(t) \leq \int_{c}^{t_{1}}(t-s)^{\alpha-1}|F(s)| d s+e(t)+(1-\lambda) \\
\cdot \lambda^{\lambda /(1-\lambda)} \int_{t_{1}}^{t}(t-s)^{\alpha-1} s^{\gamma-1} m^{\lambda /(\lambda-1)}(s) \\
\cdot h^{1 /(1-\lambda)}(s) d s+\int_{t_{1}}^{t}(t-s)^{\alpha-1} s^{\gamma-1} m(s) x(s) d s .
\end{gathered}
$$

Integrating inequality (42) from $t_{1}$ to $t$ and interchanging the order of integration one can easily obtain

$$
\begin{aligned}
x(t) \leq & x\left(t_{1}\right)+\int_{t_{1}}^{t} g_{+}(s) d s \\
& +\int_{t_{1}}^{t} \int_{t_{1}}^{u}(u-s)^{\alpha-1} s^{\gamma-1} m(s) x(s) d s d u \\
& +\int_{t_{1}}^{t} \int_{c}^{t_{1}}\left(t_{1}-s\right)^{\alpha-1}|F(s)| d s d u .
\end{aligned}
$$

Interchanging the order of integration in second integral we have

$$
\begin{aligned}
x(t) \leq & x\left(t_{1}\right)+\int_{t_{1}}^{t} g_{+}(s) d s \\
& +\frac{t}{\alpha} \int_{t_{1}}^{t}(t-s)^{\alpha-1} s^{\gamma-1} m(s) x(s) d s \\
& +\int_{t_{1}}^{t} \int_{c}^{t_{1}}\left(t_{1}-s\right)^{\alpha-1}|F(s)| d s d u .
\end{aligned}
$$

The rest of the proof is similar to that of Theorem 3 and hence is omitted.

Example 9. Let $p>1,0<\alpha=1-1 / 2 p<1, \alpha=\gamma$, and $q=p /(p-1)$. Clearly,

$$
\begin{aligned}
p(\alpha-1)+1 & =p(\gamma-1)+1=p\left(1-\frac{1}{2 p}-1\right)+1 \\
& =\frac{1}{2}>0, \\
\theta & =p(\alpha+\gamma-2)+1=0 .
\end{aligned}
$$

Let the functions $e(t)=t \sin t$ and $f(t, x)=t^{\gamma-1} h(t) x^{\lambda}$, where $0<\lambda<1, h \in C\left(\mathbb{R}^{+}, \mathbb{R}^{+}\right)$with $h(t)=m(t), \int^{\infty} s^{q} h^{q}(s) d s<$ $\infty$, and

$$
\limsup _{t \rightarrow \infty} \frac{1}{t} \int_{t_{1}}^{t}(t-s)^{\alpha-1} s^{\gamma-1} h(s) d s<\infty .
$$

Condition (39) is also fulfilled. Thus, all conditions of Theorem 8 are satisfied and hence every nonoscillatory solution $x$ of (37) satisfies lim $\sup _{t \rightarrow \infty}(|x(t)| / t)<\infty$.

Finally, we consider the integral equation

$$
\begin{array}{r}
x(t)=e(t)+\int_{c}^{t}(t-s)^{\alpha-1} k(t, s) f(s, x(s)) d s, \\
c>1, \alpha \in(0,1) .
\end{array}
$$

Now we give sufficient conditions for the boundedness of any nonoscillatory solution of (47).

Theorem 10. Let $0<\lambda<1$ and let condition (ii) hold and suppose that $p>1, q=p /(p-1), 0<\alpha<1$, and $\gamma=$ $2-\alpha-1 / p, p(\alpha-1)+1>0$, and $p(\gamma-1)+1>0$,

$$
\begin{aligned}
& \int_{t_{1}}^{\infty} m^{q}(s) d s<\infty, \\
& \limsup _{t \rightarrow \infty} g_{-}(t)<\infty, \\
& \liminf _{t \rightarrow \infty} g_{+}(t)>-\infty,
\end{aligned}
$$

where $g(t)$ is defined as in (5) for any $t_{1} \geq c$. If $x$ is a nonoscillatory solution of (47), then $x$ is bounded.

Proof. Let $x$ be an eventually positive solution of (47). We may assume that $x(t)>0$ for $t \geq t_{1}$ for some $t_{1} \geq c$. We let $F(t)=f(t, x(t))$. In view of (ii) we may then write

$$
\begin{aligned}
& x(t) \\
& \leq \int_{c}^{t_{1}}(t-s)^{\alpha-1}|F(s)| d s+e(t) \\
& \quad+\int_{t_{1}}^{t}(t-s)^{\alpha-1} s^{\gamma-1}\left[h(s) x^{\lambda}(s)-m(s) x(s)\right] d s \\
& \quad+\int_{t_{1}}^{t}(t-s)^{\alpha-1} s^{\gamma-1} m(s) x(s) d s
\end{aligned}
$$

or

$$
\begin{aligned}
x(t) \leq & \int_{c}^{t}(t-s)^{\alpha-1}|F(s)| d s+g_{+}(t) \\
& +\int_{t_{1}}^{t}(t-s)^{\alpha-1} s^{\gamma-1} m(s) x(s) d s .
\end{aligned}
$$

The rest of the proof is similar to that of Theorem 3 and hence is omitted. 
Example 11. Let $p>1,0<\alpha=1-1 / 2 p<1, \alpha=\gamma$, and $q=p /(p-1)$. Clearly,

$$
\begin{aligned}
p(\alpha-1)+1 & =p(\gamma-1)+1=p\left(1-\frac{1}{2 p}-1\right)+1 \\
& =\frac{1}{2}>0, \\
\theta & =p(\alpha+\gamma-2)+1=0 .
\end{aligned}
$$

Let the functions $e(t)=\sin t$ and $f(t, x)=t^{\gamma-1} h(t) x^{\lambda}$, where $0<\lambda<1, h \in C\left(\mathbb{R}^{+}, \mathbb{R}^{+}\right)$with $h(t)=m(t), \int^{\infty} h^{q}(s) d s<\infty$, and

$$
\limsup _{t \rightarrow \infty} \int_{t_{1}}^{t}(t-s)^{\alpha-1} s^{\gamma-1} h(s) d s<\infty
$$

Condition (49) is also fulfilled. Thus, all conditions of Theorem 10 are satisfied and hence every nonoscillatory solution $x$ of (37) is bounded.

Similar reasoning to that in the sublinear case guarantees the following theorems for the integrodifferential equations (37) and (47) when $\lambda=1$.

Theorem 12. Let $\lambda=1$ and the hypotheses of Theorems 8 and 10 hold with $m(t)=h(t)$. Then the conclusion of Theorems 8 and 10 holds.

We may note that results similar to Theorem 4 can be obtained for (37) and (47). The details are left to the reader.

\section{General Remarks}

(i) The results of this paper are presented in a form which is essentially new and it can also be employed to investigate the asymptotic and oscillatory behavior of certain integrodifferential equations of higher order $\alpha \in(n-1, n), n \geq 1$. The details are left to the reader.

(ii) It would be of interest to study (1) when $f$ satisfies condition (iii) with $\lambda>1$.

\section{Competing Interests}

The authors declare that they have no competing interests.

\section{References}

[1] D. Bleanu, J. A. T. Machado, and A. C.-J. Luo, Fractional Dynamics and Control, Springer, New York, NY, USA, 2012.

[2] V. Lakshmikantham, S. Leela, and J. Vaaundhara Dev, Theory of Fractional Dynamic Systems, Cambridge Scientific Publishers, 2009.

[3] A. A. Kilbas, H. M. Srivastava, and J. J. Trujillo, Theory and Applications of Fractional Differential Equations, vol. 204 of North-Holland Mathematics Studies, Elsevier, Amsterdam, The Netherlands, 2006.

[4] K. S. Miller and B. Ross, An Introduction to the Fractional Calculus and Fractional Differential Equations, John Wiley \& Sons, New York, NY, USA, 1993.
[5] Q.-H. Ma, J. Pecaric, and J.-M. Zhang, "Integral inequalities of systems andthe estimate for solutions of certain nonlinear two-dimensional fractional differential systems," Computers \& Mathematics with Applications, vol. 61, no. 11, pp. 3258-3267, 2011.

[6] I. Podlubny, Fractional Differential Equations, vol. 198 of Mathematics in Science and Engineering, Academic Press, San Diego, Calif, USA, 1999.

[7] A. P. Prudnikov, Z. A. Brychkov, and O. I. Marichev, "Integral and series," in Elementary Functions, vol. 1, Nauka, Moscow, Russia, 1981 (Russian).

[8] S. G. Samko, A. A. Kilbas, and O. I. Marichev, Fractional Integrals and Derivatives: Theory and Applications, Gordon and Breach Science, New York, NY, USA, 1993.

[9] M. Bohner, S. Grace, and N. Sultana, "Asymptotic behavior of nonoscillatory solutions of higher-order integro-dynamic equations," Opuscula Mathematica, vol. 34, no. 1, pp. 5-14, 2014.

[10] S. R. Grace and A. Zafer, "Oscillatory behavior of integrodynamic and integral equations on time scales," Applied Mathematics Letters, vol. 28, pp. 47-52, 2014.

[11] S. R. Grace, J. R. Graef, and A. Zafer, "Oscillation of integrodynamic equations on time scales," Applied Mathematics Letters, vol. 26, no. 4, pp. 383-386, 2013.

[12] S. R. Grace, J. R. Graef, S. Panigrahi, and E. Tunc, "On the oscillatory behavior of Volterra integral equations on timescales," Panamerican Mathematical Journal, vol. 23, no. 2, pp. 35-41, 2013.

[13] S. R. Grace, R. P. Agarwal, P. J. Y. Wong, and A. Zafer, "On the oscillation of fractional differential equations," Fractional Calculus and Applied Analysis, vol. 15, no. 2, pp. 222-231, 2012.

[14] G. H. Hardy, I. E. Littlewood, and G. Polya, Inequalities, Cambridge University Press, Cambridge, UK, 1959. 


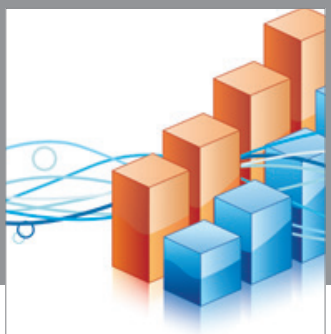

Advances in

Operations Research

vatem alat4

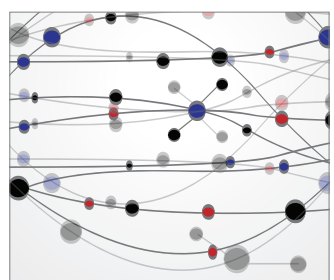

\section{The Scientific} World Journal
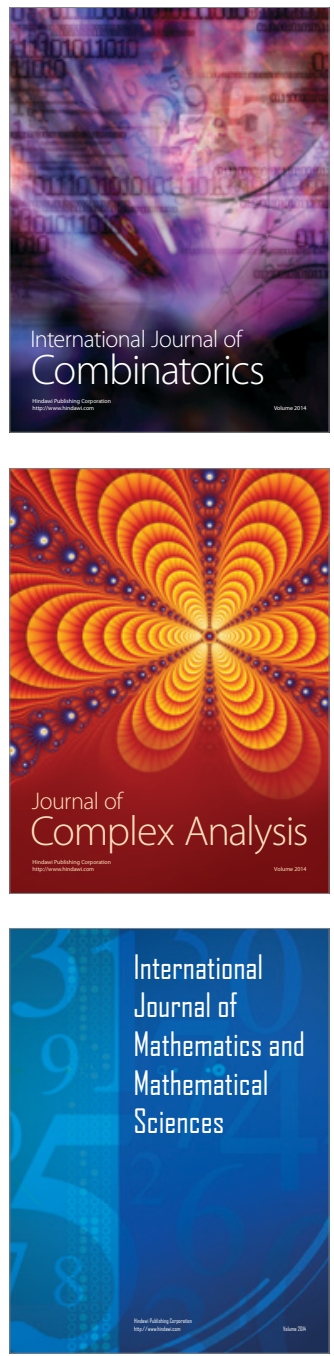
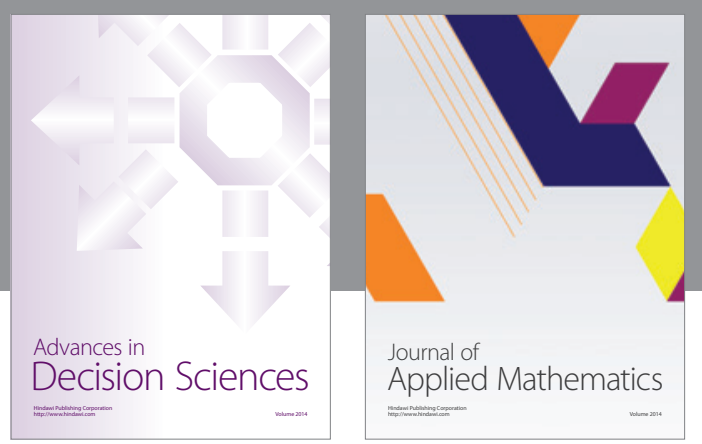

Algebra

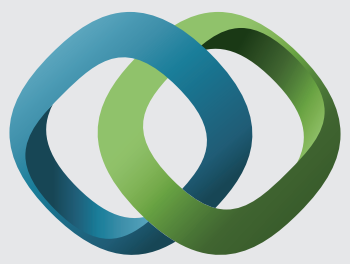

\section{Hindawi}

Submit your manuscripts at

http://www.hindawi.com
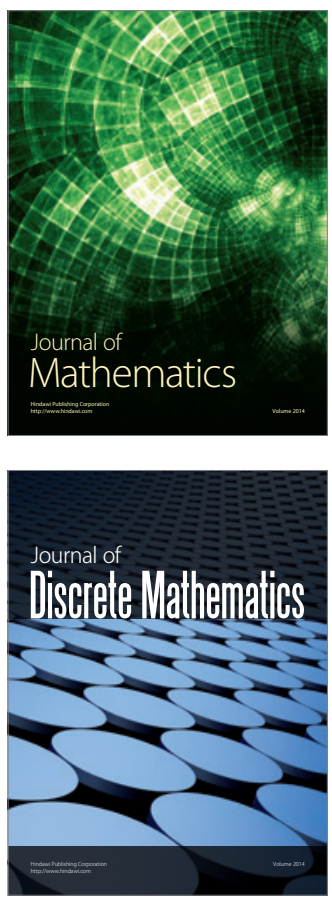

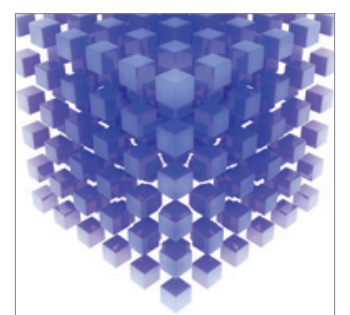

Mathematical Problems in Engineering
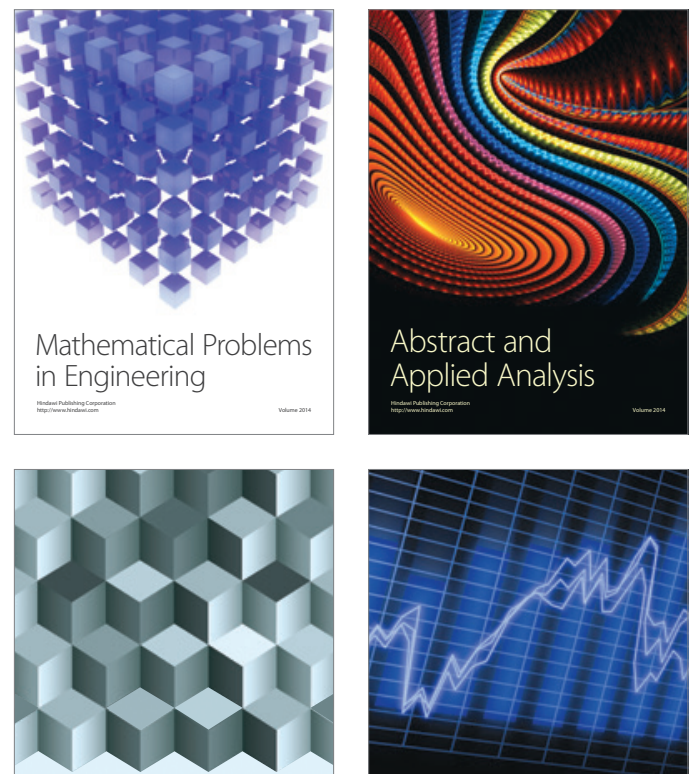

Journal of

Function Spaces

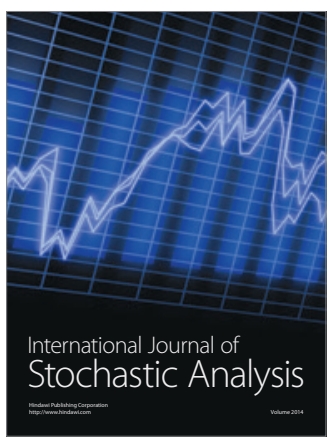

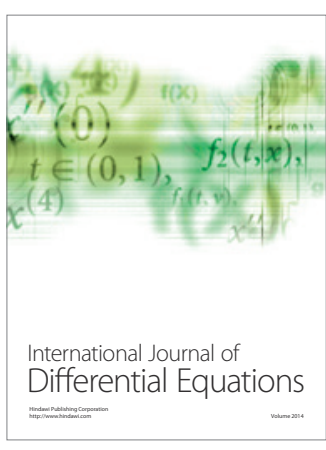
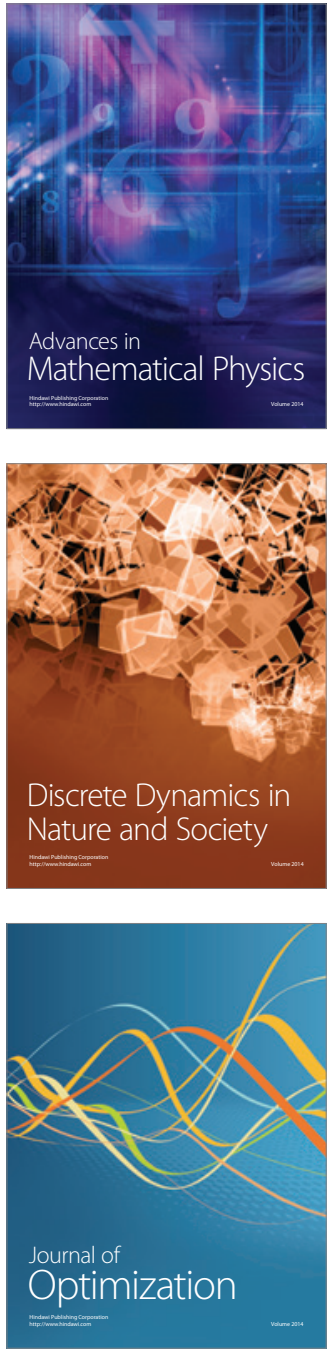